BIO Web of Conferences 1, 00102 (2011)

DOI: $10.1051 /$ bioconf $/ 20110100102$

(C) Owned by the authors, published by EDP Sciences, 2011

\title{
Audio-tactile events can improve the interlimb coordination in Juggling
}

\author{
G. Zelic ${ }^{*}$, D. Mottet ${ }^{*}$, J. Lagarde* \\ (*) Movement To Health, EuroMov, Montpellier 1 University, 700 Av. Pic Saint Loup 34090 \\ Montpellier, France
}
E-mail: gregory.zelic@univ-montp1.fr,denis.mottet@univ-montp1.fr,julien.lagarde@univ- montpl.fr

\begin{abstract}
Elementary reactive behavior can be improved in the case of cross-modal sensory interactions thanks to underlying multisensory integration mechanisms. Can this benefit be generalized to a challenging ongoing perceptive-motor performance? We choose a juggling task to examine this question. A central issue wellknown in juggling lies in establishing and maintaining a specific temporal coordination among balls, hands, eyes and posture. Here, we tested whether providing additional timing information about the balls and hands motions by using external sound and tactile periodic stimulations improved the performance of jugglers. Results indicated that specific combination of auditory and tactile metronome led to a decrease of the spatiotemporal variability of the juggler's performance. A contrario, degraded performances were obtained in the case of unimodal tactile metronome. We discuss these results in view of the understanding of the neuronal integration process implied in the audio-tactile interaction, and considering the well-known gating effect of movement on vibrotactile perception.
\end{abstract}

\section{Introduction}

A recent part of research has evidenced the efficiency of multimodal integration through the improvement of detection, reaction, and discrimination $[1,2]$. However little is known about how cross-modal environments can contribute to the ongoing coordination of limbs. We examine here multisensory processes involved when perception and action come together in ongoing coordination. The coordination of movements has a pervasive functional role in elementary behaviors as in daily actions [3, 4]. Such coordination requires dynamic and reciprocal information exchanges between brain, body and the environment. Though intrinsically multisensory (i.e. combining vision, audition, touch, and proprioception), interlimb coordination is in many cases very dependent upon the use of vision. Here we are interested in the advantage audio-tactile cues, which leave the optical array invariant, may provide to coordination.

Detection of tactile events is reduced during the execution of movements, a gating effect, which varies during the time course of movement $[5,6]$. In the present study the tactile detection was not achieved without a functional relation to the movement produced; rather the stimuli endorsed the role of carrying over relevant information to enhance an ongoing coordination.

We selected in the present study the so-called three balls cascade juggling trick denoted juggling in the following. Juggling is mainly characterized by a $1: 3$ frequency ratio between the hand movement and a ball cycle. This notably entails forming and maintaining multi-frequency relations among movements of hands, eyes and posture [7, 8].

Sensory stimulation has been evidenced to be able to increase the robustness of elementary coordination against internal biological noise or external perturbation [9]. Such external timing information should be beneficial to the juggler. However, a simple auditory metronome failed to stabilize juggling, which indicates that the multi-frequency nature of the juggling calls for more than a mere metronome.

In the present study, our prediction that one can stabilize the juggling coordination with adequate external sensory cues was tested using periodic audiotactile stimuli, respectively presented at the ears and at the wrists, and providing specific tempo.

This is an Open Access article distributed under the terms of the Creative Commons Attribution-Noncommercial License 3.0, which permits unrestricted use, distribution, and reproduction in any noncommercial medium, provided the original work is properly cited. 
Table 1: the six experimental conditions

\begin{tabular}{|c|c|c|c|c|c|}
\hline \multicolumn{5}{|c|}{ Metronomes characteristics } & \multirow{2}{*}{ Experimental } \\
Metronome \\
Sensory & \multirow{2}{*}{$\begin{array}{c}\text { Structure } \\
\text { Modality }\end{array}$} & \multirow{2}{*}{$\begin{array}{c}\text { Auditory } \\
\text { frequency }\end{array}$} & frequency & phasing & Abbreviation \\
\hline Audio-tactile & Simple & $\Omega$ Sall & $\Omega$ hand & $\phi=0$ & Multi. Simp. \\
\hline Audio-tactile & Simple & $\Omega$ ball & $\Omega$ hand & $\phi=\pi$ & Multi. Anti. \\
\hline Audio-tactile & Double & $2 * \Omega$ ball & $2 * \Omega$ hand & $\phi=0$ & Multi. Doub. \\
\hline Tactile & Double & - & $2 * \Omega$ hand & $\phi=0$ & Tact. Doub. \\
\hline Audio & Double & $2 * \Omega$ ball & - & & Audio. Doub. \\
\hline- & - & - & - & - & Control \\
\hline
\end{tabular}

\section{Method}

Seven adults, from 19 to 26 years of age, volunteered for this experiment. All of them have an intermediate level of juggling.

Balls and hands positions were recorded with an optical 3D motion analysis system at a sampling frequency of $100 \mathrm{~Hz}$. The vibrotactile metronome consisted in $80 \mathrm{~ms}$ square wave pulses (vibration carrier frequency: $100 \mathrm{~Hz}$ ) strapped on the ventral part of each wrist. The auditory metronome consisted in 80 ms square wave pulses (tone carrier frequency: 300 $\mathrm{Hz}$ ) sent to auricles.

Participants were instructed to start juggling after the metronome started; no instructions to synchronize with the stimuli were given.

\subsection{Conditions}

For each participant, the tactile and auditory metronomes were set up to match the preferred hands and balls frequencies ( $\Omega$ hand and $\Omega$ ball). Since balls and hands frequencies are core to the multiple component coordination in juggling, we chose to associate each sensory modality to one of these main components.

Experimental conditions are presented in the next table (Table 1). We compared three audio-tactile metronomes and two unimodal tactile and auditory metronomes. The frequencies of tactile and auditory metronomes were adjusted to be respectively equal to (simple mode: Multi. Simp., Multi. Anti.) or twice (double mode: Multi. Doub., Tact. Doub., Audio. Doub.) the frequency of hands and of balls measured in the preliminary trial. We will borrow this "simple" and "double" terminology for the remainder of this article.

We added a "simple" audio-tactile metronome with antiphase tactile stimuli on each wrist (Multi. Anti.) to reproduce the natural relative phase between the hands movement in juggling.

\section{$1.2 \quad$ Variables}

We measured the variability of juggling with spatial and temporal dependent variables. Throwing behavior was described by tossing angles and velocities, which fully determine the trajectories of the balls.

We thus examined:

- The time of the ball in flight (Tf) between a throw and the consecutive catch by the other hand;

- The well-known K-ratio, which is the percentage of a cycle with the ball in hand;

- The vertical and the horizontal distance between two consecutive throws, thus involving both hands.

We estimated the behavioral variability for each participant for a given variable by calculation of the standard deviation within a trial.

\subsection{Synchronization to metronome}

We computed the average difference between the observed hand frequency and the frequency of the metronome. Subsequently we analyzed the correlation between the absolute values of these differences and the variability of the juggling variables.

We computed the circular variance, and the dwell time of the relative phase between a hand and the metronome. The dwell time was defined as the proportion of points for which the derivative of the relative phase was smaller than a limit value $(0.1,0.15$, 0.2 , and 0.25 ).

\subsection{Statistical analysis}

We used an analysis of variance (ANOVA) with repeated measures to assess the significance of the effects of the different metronomes. Because of the sphericity constraint we report all $p$ values after Huynh-Feldt correction. The Fisher's least significant difference (LSD) post hoc-test was used when applicable.

To take into account the high level of interparticipant variability, we used a control condition without any periodic sensory stimulation. For each participant, the standard deviation in the control 
condition was subtracted to the mean variability reached in the other experimental conditions.

A mean comparison t-test was used to examine significant differences of variability of each data set from the zero level, corresponding to the baseline level of variability measured in the control condition.

\section{Results}

The ANOVA on the averages of variability of the velocity at throws indicated an effect of the metronome condition (Figure $1 ; \mathrm{F}(4,24)=3.03, \mathrm{P}=0.037$ ). Posthoc comparisons showed that the antiphase audiotactile condition (Multi. Anti.) reduced the variability of the velocity at throws when compared to both unimodal conditions (Tact. Doub. and Audio. Doub.). Moreover the in-phase audio-tactile condition (Multi. Simp.) reduced the variability of the velocity at throws when compared to the unimodal tactile condition (Tact. Doub.).

In addition, the mean comparison t-test showed that the variability realized in the unimodal tactile condition (Tact. Doub.) was significantly larger than the zero variability baseline $(\mathrm{t}(30)=2.1273, \mathrm{p}<.05)$.

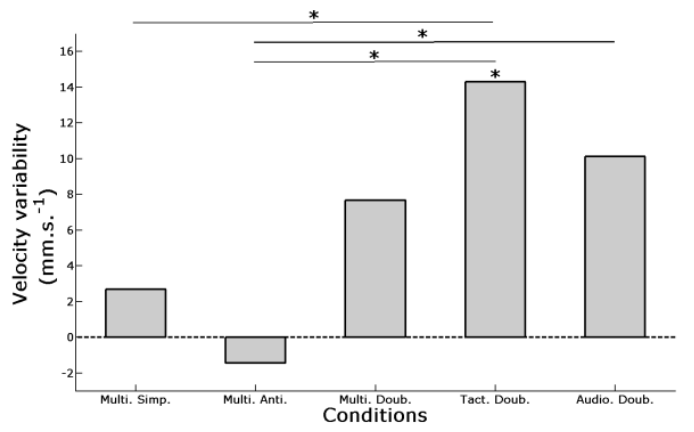

Figure 1: Throws velocity variability for each metronome condition. The group average after subtraction of the baseline variability is presented. The stars on the top of the horizontal lines indicate significant differences between two conditions. The star upper the distribution bar of the Tactile Double condition indicates a significant difference with the baseline variability.

The ANOVA applied on the averages of variability of the K-ratio was inconclusive.

The ANOVA on the variability of the balls flight time (Tf) revealed a significant effect of the metronome condition $(\mathrm{F}(4,24)=3.6, \mathrm{p}<.05)$. Post-hoc comparisons showed that the antiphase audio-tactile condition (Multi. Anti.) reduced more the variability of the ball flight duration than the in-phase audio-tactile simple condition (Multi. Simp.) and the unimodal ones
(Tact. Doub. and Audio. Doub.). In addition, the audiotactile double-metronome (Multi. Doub.) generated a stronger reduction of variability of the ball flight time than the unimodal tactile metronome (Tact. Doub.).

The ANOVA showed a significant effect of the metronome condition on the variability of the vertical distance between two consecutives throws (BTy) (Figure 2; $\mathrm{F}(4,24)=3.62, \mathrm{p}<.02)$. Post-hoc comparison revealed that the antiphase audio-tactile condition (Multi. Anti.) lowered variability in comparison to the in-phase audio-tactile (Multi. Simp.) and the unimodal tactile (Tact. Doub.) conditions. In addition the audio-tactile simple-metronome (Multi. Simp.) induced an increased variability compared to the audio-tactile double-metronome (Multi. Doub.) and the unimodal audio one (Audio. Doub.).

In addition, the mean comparison t-test showed that the mean of the vertical variability reached in the antiphase audio-tactile condition (Multi. Anti.) was significantly below the zero level, that was, the variability baseline corresponding to the control condition $(\mathrm{t} 30=-2.2371, \mathrm{p}<.05)$.

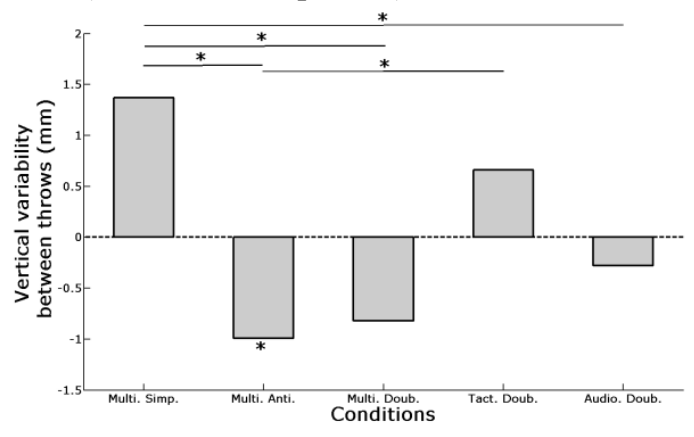

Figure 2: Variability of the vertical distance between consecutives throws for each metronome condition. Here the group average after subtraction of the baseline variability is shown.. The star below the distribution bar of the antiphase audio-tactile condition indicates a significant difference with the baseline variability.

\subsection{Entrainment by the metronome}

The ANOVA on the differences between the frequency of the motion of the hand of the juggler and the frequency of the metronome was not conclusive. A Pearson's correlation analysis indicated that the variability of the juggling pattern significantly increased with the calculated frequency difference. This was evidenced for the ball flight time $(\mathrm{R} 2=12 \%$, $\mathrm{p}<.05)$, the velocity at throws $(\mathrm{R} 2=25 \%, \mathrm{p}<.01)$, and the vertical distance between throws $(\mathrm{R} 2=56 \%, \mathrm{p}$ $<.001)$. Moreover, we also found that the variability of the vertical distance between throws was more related to the frequency difference for the antiphase audio- 
tactile metronome $(\mathrm{R} 2=40 \%, \mathrm{p}<0.001)$ than for the other conditions ( $\mathrm{R} 2=20 \%$ to $28 \%, \mathrm{p}<0.01$ ).

\section{Conclusion}

We found that a tactile metronome, presented synchronously at the wrists, increased the variability of the velocity at the throws when compared to the baseline variability measured in the absence of a metronome. This detrimental effect of the tactile metronome on the stability of the juggling reveals a neat difficulty to integrate the information conveyed by a tactile metronome within an ongoing coordination. This result is in agreement with the hypothesis of a detrimental effect of tactile stimuli on the control of movements, based on the so-called gating effect.

We found that audio-tactile metronomes generally positively influenced the juggling performance more than the audio or the tactile metronome alone. Intuitively, this makes sense since the multimodal metronome provided an enriched environment. Each one brings two distinct tempos sustained by two different sensory pathways, along with specific frequency ratio and relative phasing between them, and in one case, a relative phasing between the vibrations applied at the wrist. We assume that ensemble of properties may have collectively contributed to strengthen, or stabilize, the perception- action coordination underlying the relation between the hands and the balls.

We emphasize that a multi-modal effect was found for the variability of the flight duration between a throw and a catch. The minimization of the variability of the flight duration in skilled jugglers was interpreted as the signature of a global variable of coordination. Differently the tactile detrimental effect was obtained for the velocity at throws, which represents a local variable in the juggling coordination. We suggest that the audio-tactile stimulations can overcome the tactile detrimental effect, not by acting at the level of the coordination of the throwing movements but by ordering the global pattern of coordination, which requires a tight coupling between the movement of the hands and visual information pick up about the motion of the balls.

We evidenced that the antiphase audio-tactile metronome generally decreased the variability of juggling behavior in comparison to the two other audio-tactile conditions. What is of particular interest is that the audio-tactile simple metronome differed only by the relative phasing between the tactile events. Moreover the antiphase audio-tactile metronome reduced the variability of the vertical distance between throws in comparison to the baseline corresponding to the control condition. This directly evidenced the enhancement of the juggling performance caused by specific parameters of an audio-tactile metronome. Thus matching the phasing of the tactile stimulations with the actual phasing of the movement of the hands reduced the variation of distance between successive projection points of left and right hands. This clearly demonstrated the key role relative phasing can play in the effective integration of audio-tactile cues with an interlimb coordination pattern, and agreed with the assumption that the degree to which information can be useful is the degree to which it can be integrated within the intrinsic behavioral dynamics.

This research was supported by SKILLS, an Integrated Project (FP6-IST contract \#035005) of the Commission of the European Community.

\section{References}

M.A. Meredith, B.E. Stein. Interactions among converging sensory inputs in the superior colliculus, Science, 221(4608), pages. 389-91, 1983

H.F. Sperdin, C. Cappe, M.M. Murray. The behavioral relevance of multisensory neural response interactions. Front Neurosci,. 4, pages. 9, 2010

J.A.S. Kelso. Dynamic patterns: The self-organization of brain and behavior, The MIT Press., 1995

M.T. Turvey. Coordination, Am Psychol., 45(8), pages. 93853, 1990

M. Voss, J.N. Ingram, P. Haggard, D.M. Wolpert. Sensorimotor attenuation by central motor command signals in the absence of movement, Nat Neurosci., 9(1), pages. 267,2006

G. Juravle, H. Deubel, H.Z. Tan, C. Spence. Changes in tactile sensitivity over the time-course of a goal-directed movement, Behav Brain Res, 208(2), pages. 391-401, 2010

P.J. Beek, M.T. Turvey. Temporal patterning in cascade juggling, J Exp Psychol Hum Percept Perform, 18(4), pages. 934-47, 1992

R. Huys, A. Daffertshofer, P.J. Beek. Multiple time scales and subsystem embedding in the learning of juggling, Hum Mov Sci., 23(3-4), pages. 315-36? 2004

P.W. Fink, P. Foo, V.K. Jirsa, J.A. Kelso. Local and global stabilization of coordination by sensory information, Exp Brain Res, 134(1), pages. 9-20, 2000 